\title{
An electronic system for the measurement electrochemical corrosion type
}

\begin{abstract}
The present work shows the development of a low-cost device to measure the type of electrochemical corrosion, using the electrochemical noise measurement technique, which improves the accuracy concerning commercial measurement devices by using high precision circuits, anti-aliasing filters, and a faraday cage to compensate the intrinsic noise level of the system. It was validated using three dummy cells with three different resistance values, $100 \Omega, 10 \mathrm{~K} \Omega$, and $1 \mathrm{M} \Omega$, which simulate the impedance of the electrolyte on the noise levels of the electronics modules. To validate the performance of the anti-aliasing filters used in the system, three different acquisition frequencies of $1 \mathrm{~Hz}, 100 \mathrm{~Hz}$, and $1 \mathrm{KHz}$ were used. The better results obtained for the acquisition frequency of $1 \mathrm{KHz}$ in which the $\sigma \mathrm{i}$ varies from $140 \mathrm{pA}$ for the $100 \Omega$ dummy cell to $200 \mathrm{pA}$ for the $1 \mathrm{M} \Omega$ dummy cell, the $\sigma \mathrm{V}$ varies from $120 \mu \mathrm{V}$ for the $100 \Omega$ dummy cell to $200 \mu \mathrm{V}$ for the $1 \mathrm{M} \Omega$ dummy cell. Furthermore, the system was tested using a cell with three identical aluminum electrodes, submerged in a solution of distilled water with $3.5 \% \mathrm{NaCl}$ for 6.5 hours. The results from this test show a general and localized corrosion in the form of pitting that characterizing this type of experiment.
\end{abstract}

Keywords: electrochemical noise, corrosion, pitting, intrinsic noise, antialiasing filter
Volume 6 Issue 4 - 2020

\section{Fuentes Castro JA, Laria Menchaca J, Ibarra Martínez S, Castán Rocha JA, Terán Villanueva JD \\ Department of Facultad de Ingeniería" Arturo Narro Siller", Universidad Autónoma de Tamaulipas, México}

\begin{abstract}
Correspondence: Laria Menchaca J, Department of Facultad de Ingeniería" Arturo Narro Siller", Universidad Autónoma de Tamaulipas, Permanent Postal address: Miguel Cortez 109, Col. Petroquímicas, C.P. 89327,Tampico, Tamaulipas, México, Email jlaria@docetes.uat.edu.mx
\end{abstract}

Received: October 04, 2020 | Published: December 2I, 2020

\section{Introduction}

In the industry, it is essential to assess the corrosion level of the production systems regarding their components. However, it is of interest the parts and components for which the corrosion can cause accidents or the unscheduled stoppage of a plant, which usually incurs in a significant economic loss. It is in this context that the most used techniques for the corrosion measurement are the electrochemical techniques, ${ }^{1}$ particularly: the linear polarization resistance (LPR), the electrochemical impedance spectroscopy (EIS), and the electrochemical noise measurement (ENM). Although all of them have shown excellent results for monitoring the corrosion, the ENM showed better capabilities, because it does not need to disturb the system and allows determining the type of corrosion that is occurring in the metal. ${ }^{2}$

Lately, the ENM has been gradually accepted for the study of corrosion processes. However, it is required a great amount of research to fully understand its interpretation. ${ }^{3}$

In this context, there is evidence that many ENM has errors in their lectures caused by the absence of the proper filters; this is showed by Wouter et al. ${ }^{4}$ who carried out a round-robin test in seventeen laboratories. In that study, each laboratory used a standard protocol, in which the electrochemical noise measurements were carried out using a series of dummy cells, consisting of a star arrangement of resistors to validate the ENM equipment and determine its baseline noise performance. The study used a total of 11 brands of equipment; this includes the following commercial and general-purpose potentiostats. Gamry: Reference 600, PCI4 / 300, PCI4 / 750, ESA400, Autolab: PGSTAT 30, Solartron: SI 1287, Jaissle: ZR01 / ZU01; ACM: Gill 8 1096; Zahner: IM6; IPS: ZRA-FG / HRU-FG, as well as two custom ENM systems.

The results of that study showed a large dispersion of the data, attributed to the instrument noise, from the absence or misuse of the anti-aliasing filters in the data acquisition systems, or because of the way it is used.

These findings led the authors to conclude that most of the commercial equipment studied, is misused or inadequate to produce an accurate electrochemical noise measuring. Therefore, they emphasized that improvements are necessary for the choice of the EN measurement equipment and the need to validate measurement systems by using a dummy cell to know its baseline noise.

Regarding the lower-cost devices for measuring the electrochemical noise ${ }^{5-7}$ show the development of electrochemical noise measuring systems as an alternative to commercial equipment. Where Arellano et al. ${ }^{5}$ presented a portable device designed to measure the corrosion variables of the pitting index (PI) and the corrosion rate (CR). $\operatorname{In}^{6}$ Reid and Eden, developed a method and device for the identification of corrosion in metal objects. The method consisted in analyzing the statistical distribution of the signals generated between two electrodes made of the same material of the object to be analyzed and exposed to the same conditions. Enciso et al. ${ }^{7}$ present a system for the real-time monitoring of ENM to estimate the corrosion rate. This system uses a configuration of three electrodes and a digital-analog converter to obtain the electrochemical noise signals.

However, none of this work provides a specification of the capabilities and limitations of their measurement systems, especially those inherent in intrinsic noise.

Therefore, in this work, we propose a low-cost device to measure the type of electrochemical corrosion using the electrochemical noise measurement technique, which improves the accuracy concerning commercial measurement devices by using high precision circuits, anti-aliasing filters, and a faraday cage to compensate the intrinsic noise level of the system. It is relevant to note that no studies are showing the intrinsic noise level of low-cost electrochemical noise measurement systems for different electrochemical cell resistances. 
This document focuses on the principles of the measurement process and the validation of the measurement system and does not consider the applications of the ENM to the study of corrosion processes.

\section{Methodology}

We can find descriptions of different methodologies to determine the mechanisms, types of corrosion, and the corrosion rate using ENM in. ${ }^{3,8-12}$ However, the American Steel Testing of Materials (ASTM) introduced a standard G199 on ENM, ${ }^{13}$ providing the specifications, methods for data analysis, procedures to verify the instrumentation, and testing for ENM.

On the other hand, Wouter et $\mathrm{al} .{ }^{4}$ has shown that the ASTM G199 specifications do not ensure the correct validation of the instrumentation while proposing a protocol to validate and measure the intrinsic noise level by using a series of dummy cells. These cells are a set of three resistors in a star arrangement, as shown in Figure 1. And it is expected to produce low levels of current and potential noise, primarily the Johnson (thermal) noise of the resistors also knowing as white noise. Therefore, theoretical values for the current and potential thermal noise signal of the resistors in the cell are calculated on the assumption that there are white, and these values give the minimum level of noise that can be measured. In that case, the standard deviations of the current and potential thermal noise signal are respectively (kTf/ $\mathrm{R})^{1 / 2}$ and $(3 \mathrm{kTfR})^{1 / 2}$, where $\mathrm{k}$ is Boltzmann's constant, $\mathrm{T}$ is temperature in Kelvin, $\mathrm{R}$ is the resistance in Ohms as derived in. ${ }^{14}$

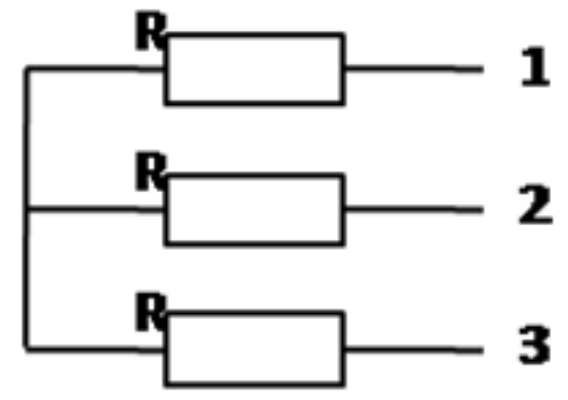

Figure I The arrangement of the dummy cell.

Therefore, we prepared three cells with three resistance values, $100 \Omega, 10 \mathrm{~K} \Omega$, and $1 \mathrm{M} \Omega$, to measure the intrinsic noise of the developed measurement system, and the impact of the impedance of the electrochemical cell on the noise levels of the current and potential measurements modules. The resistances were surface mounted resistors with $1 \%$ tolerance each and taken from the same batch.

To verify the correct functioning of the anti-aliasing filter, we made measurements with three different acquisition frequencies, $1 \mathrm{~Hz}$, $100 \mathrm{~Hz}$, and $1 \mathrm{KHz}$.

In, ${ }^{9-11,13}$ two different configurations of electrochemical cells were used for the ENM. One of them is the cell of three identical electrodes of the same material. The second configuration uses two identical electrodes of the same material and the commercial reference electrode. To verify the correct functioning of the developed system, we used a cell with three identical electrodes of aluminum $(99.8 \%$ purity) and each electrode with a surface area of $0.5 \mathrm{~cm}^{2}$. We place the electrodes parallel to each other, with $3 \mathrm{~mm}$ of separation, and they were coated with epoxy resin as shown in Figure 2. The uncovered surface of the electrodes was roughened with 600-grain sandpaper, rinsed with deionized water and dried with alcohol, and hot air under pressure. The cell was placed in desiccators for approximately 24 hours to form a passive film. We use a counting rate of $1 \mathrm{KHz}$, and the experiments were carried out at room temperature of $25^{\circ} \mathrm{C} \pm 2{ }^{\circ} \mathrm{C}$.

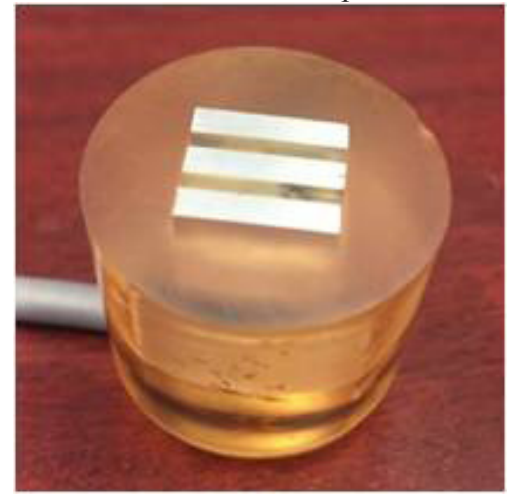

Figure 2 Cell with the aluminum electrodes used.

For the analysis of ENM, the aluminum cell was immersed six hours in a solution of distilled water with $3.5 \%$ of sodium chloride, where the potential noise and current noise dates were collected simultaneously and stored as time series. The baseline of this temporal behavior may be affected by drift. Therefore, before any treatment to the data, it is essential to perform an adjustment to eliminate the effects of this drift; thus, we used the method proposed in. ${ }^{14}$ Subsequently, we calculate the standard deviations of current noise $(\sigma i)$ and potential noise $(\sigma v)$ according to their usual definitions.

One advantage of the ENM is that it allows determining the type of corrosion befalling to the metal. To do this, we use the pitting index (PI)calculate as the standard deviation of the current noise ( $\sigma \mathrm{i})$ divided by the root mean square of the coupling current (Irms) calculated over the same sample period. ${ }^{13}$

According to the value of PI, corrosion can be classified as localized, mixed, or uniform (Table 1).

Table I Types of corrosion depending on the pitting index (PI) value

\begin{tabular}{lll}
\hline \multirow{2}{*}{ Type of corrosion } & \multicolumn{2}{l}{ PI } \\
\cline { 2 - 3 } & Min & Max \\
\hline Localized & 1 & 0.1 \\
Mixed & 0.1 & 0.01 \\
Uniform & 0.01 & 0.001 \\
\hline
\end{tabular}

\section{Results}

Figure 3 shows the block diagram of the developed ENM system, which has the previously explained cell, the electronic module for current noise measurement, the electronic module for potential noise measurement, an analog-digital converter (ADC), and a data acquisition module.

Figure 4 shows the electronic circuits of the current and potential noise measurement modules. For the current noise measurement module, we use a current to voltage converter based on the electrometric-amplifier AD549JH (U1 in Figure 4). This amplifier has a leakage current of $\pm 300 \mathrm{fA}$.; an offset current of $65 \mathrm{fA}$, an offset voltage of $\pm 260 \mu \mathrm{V}$, a voltage noise level of $92 \mathrm{nV} /(\mathrm{Hz})^{1 / 2}$ for the frequency of $10 \mathrm{~Hz}$, a current noise level of $0.22 \mathrm{fA} /(\mathrm{Hz})^{1 / 2}$ for the range of the frequencies between $0.1 \mathrm{~Hz}$ and $10 \mathrm{~Hz}$, and an input impedance of $10^{15} \Omega$. 


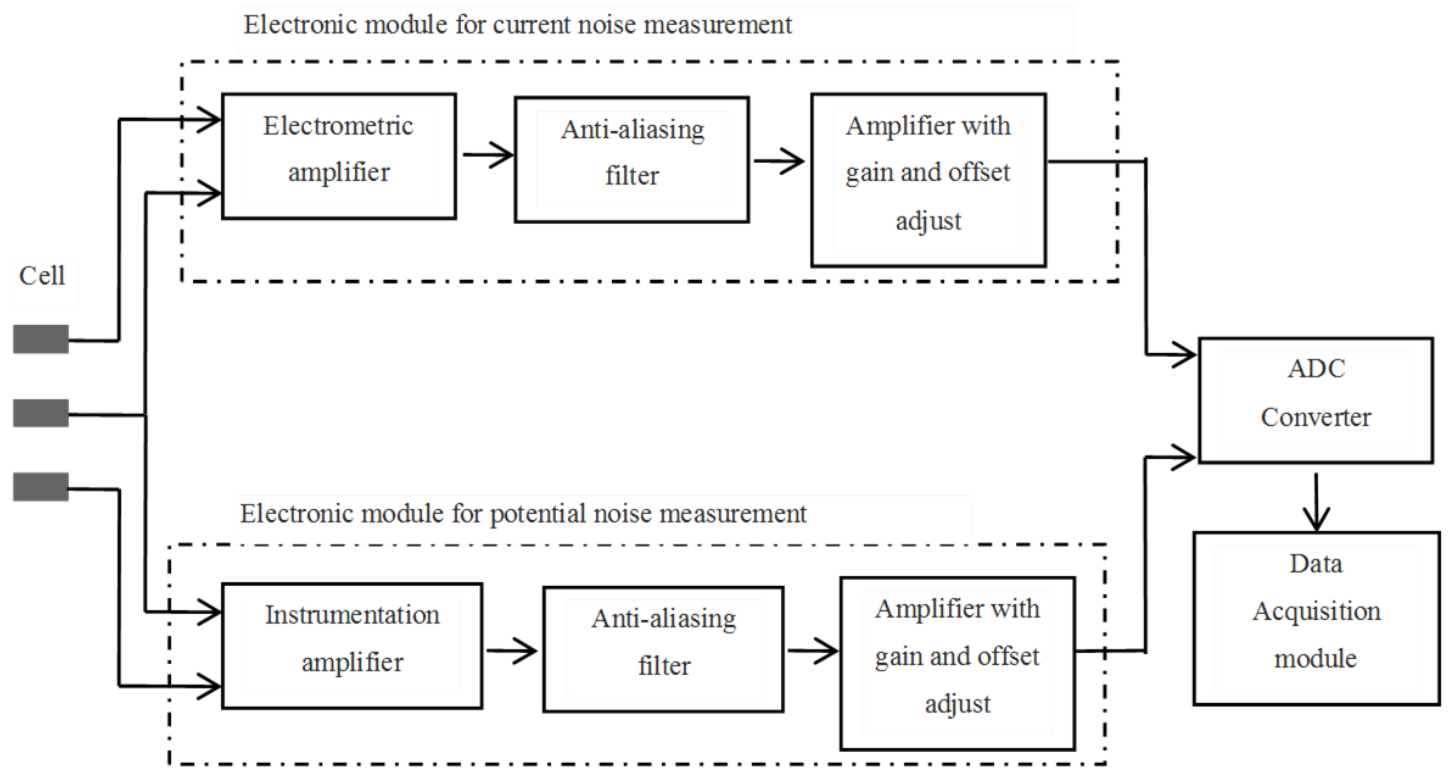

Figure 3 Block diagram of the developed ENM system.

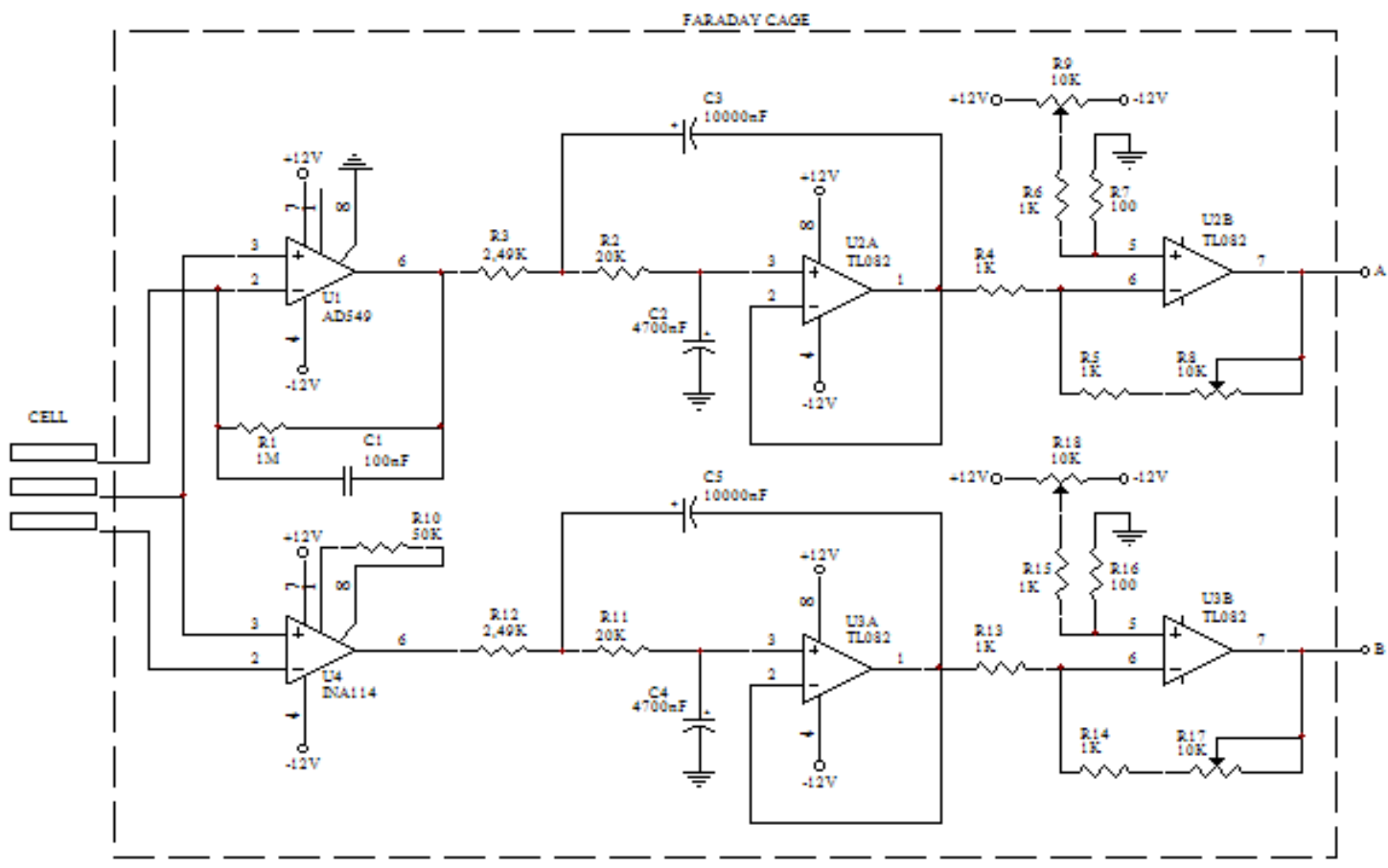

Figure 4 Electronic circuits for the current and potential noise measurement modules.

Right after the current to voltage converter, we place an active low pass filter, Bessel type, with a cut-off frequency of $10 \mathrm{~Hz}$, formed by integrated circuit $\mathrm{U} 2 \mathrm{~A}$, resistors $\mathrm{R} 2$ and $\mathrm{R} 3$ and capacitors $\mathrm{C} 2$ and $\mathrm{C} 3$. This filter has the function of anti-aliasing, which is essential for the accurate digitalization of the signal. The output of the antialiasing filter is processed by an amplifier, with gain and offset voltage adjustment (U2A, resistors R4, R5, R6, R7, and potentiometers R8 and R9). On the other hand, the electronic module for potential noise measurement was developed, based on a precision instrumentation amplifier INA114 (U4), which has an input resistance of $10^{10} \Omega$, offset voltage of $50 \mu \mathrm{V}$, current of leakage of $1 \mathrm{nA}$, a noise voltage for $10 \mathrm{~Hz}$ $11 \mathrm{nV} / \mathrm{Hz}^{1 / 2}$. The anti-aliasing filter and the amplifier, with gain and offset voltage adjustment, are like the electronic module for noise current density measurement. These two modules are placed inside a faraday cage.

Subsequently, an analog-digital converter processes the outputs of both electronic modules. Our prototype uses a multifunction card, NI USB 6009, from National Instruments, which has fourteen bits of resolution, input voltage range of $\pm 10 \mathrm{~V}$ and a maximum acquisition frequency range up to $48 \mathrm{KHz}$. We connect the multifunction card through USB to a data acquisition module, which is an industrial computer PC / 104, CPU model PFM-5401. The characteristics of the PC / 104 are as follows: AMD Geode LX800 500MHz processor, 
512M DDR400 RAM, 4 USB 2.0 ports, 4 COM ports, an Ethernet port, a parallel port, a Compact memory card of $16 \mathrm{~Gb}$ as hard drive, a power source of $+5 \mathrm{~V} / 1 \mathrm{~A}$, and runs Window XP operating system. We use Lab VIEW to write the algorithm for the data acquisition module.

This algorithm acquires the data from the two electronic modules, writing it to a file every specific time-lapse.

The used data acquisition module allows sending the data from the electronic modules to the computer using other types of communications as RS-232, RS-485, or Ethernet protocols.

To verify the intrinsic noise of the developed ENM system (Figure 3 ), the measurements of the current and the potential were made

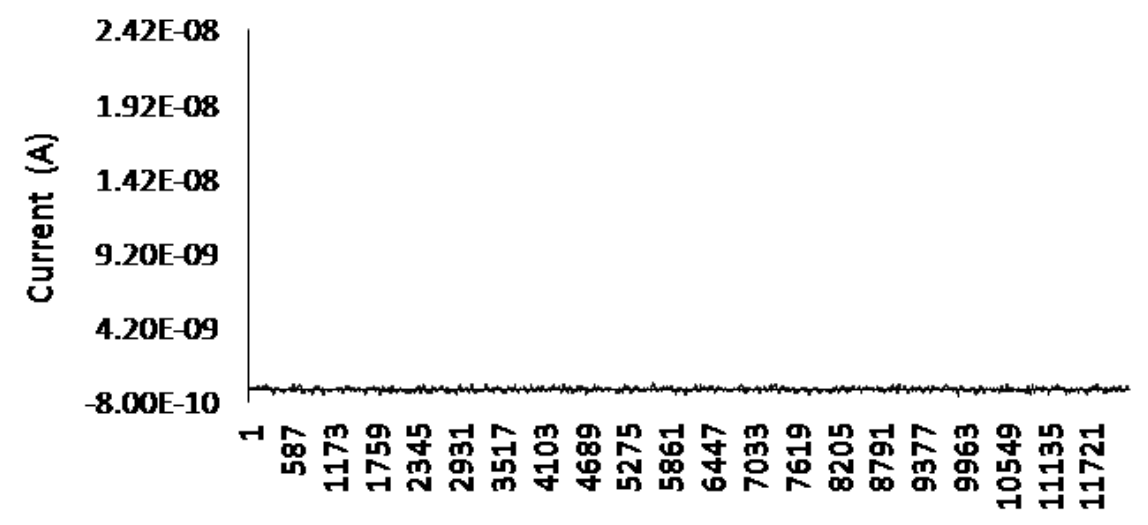

Time (s)

Figure 5 Current noise time records sampled for the $100 \Omega$ dummy cell.

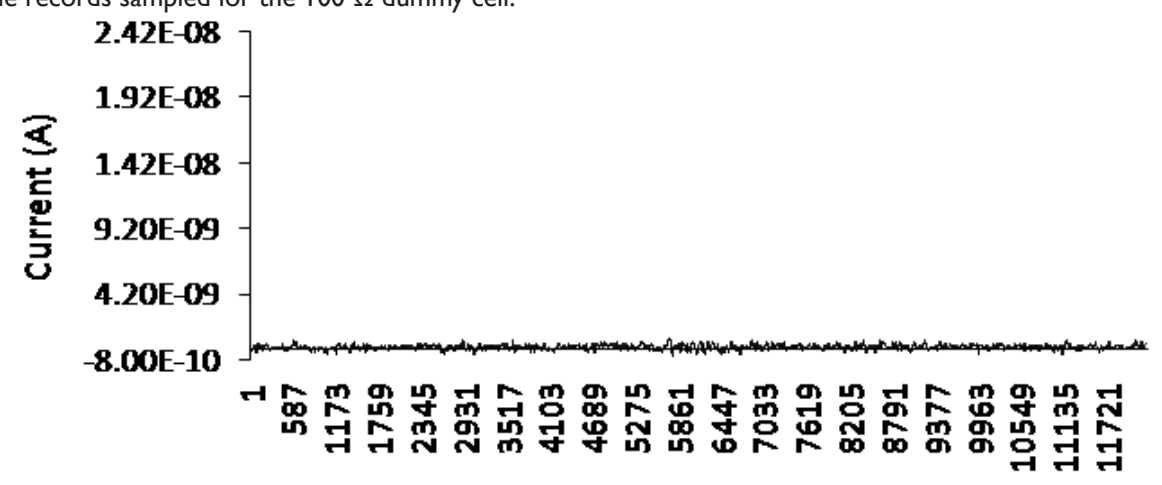

Figure 6 Current noise time records sampled for the IOK $\Omega$ dummy cell.

Time (s)

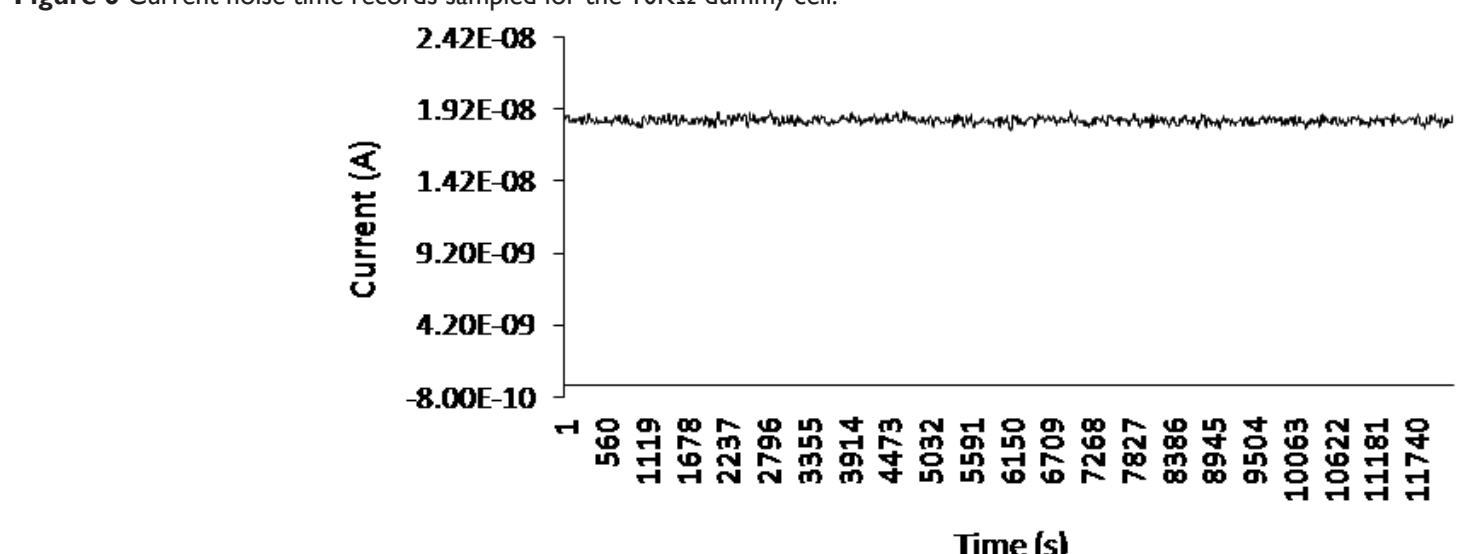

Time (s) connecting the resistive cells to ENM system, in the same way as for standard measurement. We used three different values of resistance in he cell, to simulate three different resistance of the electrolyte which current and potential noise measurements. Moreover, we used three different acquisition frequencies to verify the operation of the anti-

Figures 5-7 show the current noise time records sampled, during three and a half hours at $1 \mathrm{KHz}$ of acquisition frequency for the $100 \Omega, 10 \mathrm{~K} \Omega$, and $1 \mathrm{M} \Omega$ dummy cells, and Figures 8-10 gives the corresponding potential noise time records sampled at the same frequency. Therefore, each point in Figures 5 to 10 is the average of the 1000 samples taken during a second.

Figure 7 Current noise time records sampled for the IM $\Omega$ dummy cell. 


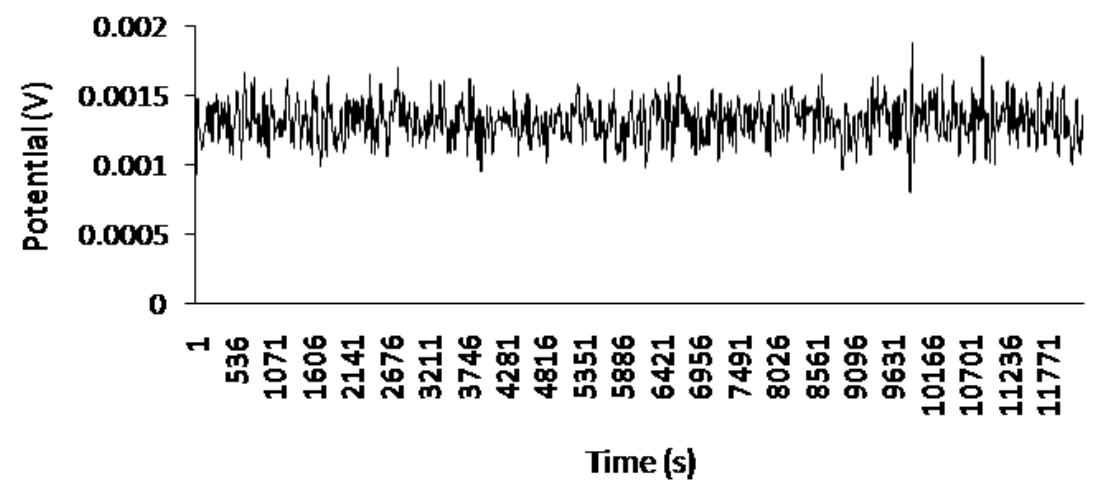

Figure 8 Potential noise time records sampled for the $100 \Omega$ dummy cell.

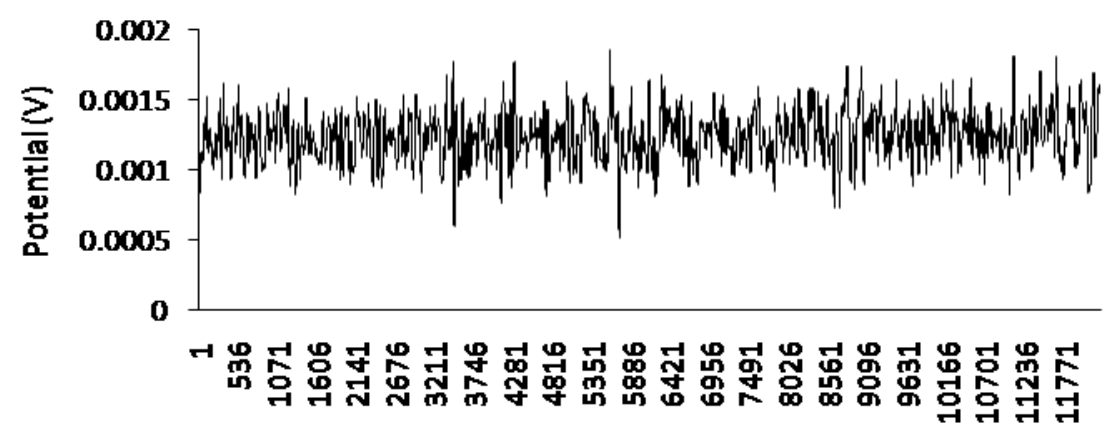

Time (s)

Figure 9 Potential noise time records sampled for the I0K $\Omega$ dummy cell.

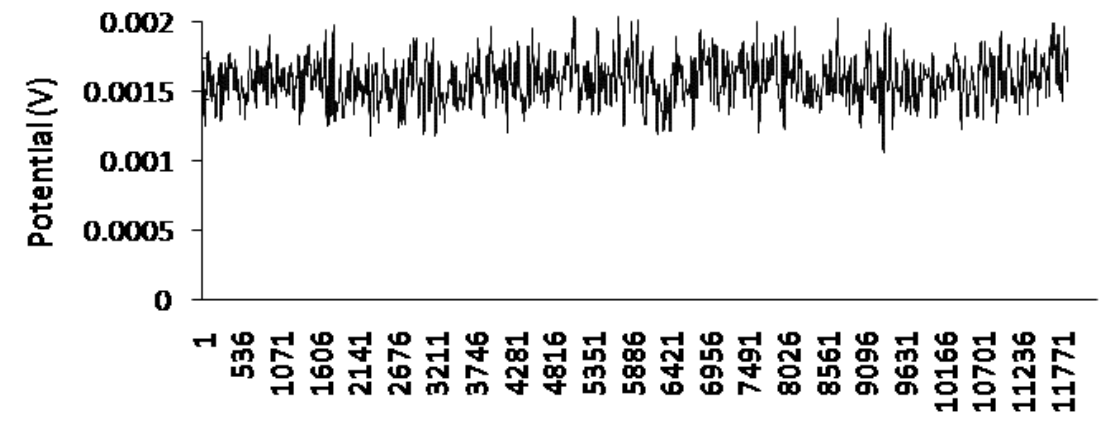

Time (s)

Figure 10 Potential noise time records sampled for the IM $\Omega$ dummy cell.

As we can see, in the time records (Figures 5-10), there is no upward or downward tendency, only the dc levels have been slightly shifted in each figure, which indicates that the results are completely dominated by the electronic noise of the developed ENM system.

This same behavior was obtained for the acquisition frequencies of $1 \mathrm{~Hz}$ and $100 \mathrm{~Hz}$.

On the other hand, Table 2 shows the average standard deviation for the current $(\sigma i)$ and potential $(\sigma \mathrm{v})$ time records and the calculated thermal noise of the resistance $(\sigma t)$ at three different acquisition frequencies for each dummy cell.

The behavior of the values in Table 2 shows that for the three resistive cells the smallest $\sigma i$ and $\sigma \mathrm{v}$ were obtained for the acquisition frequency of $1 \mathrm{KHz}$. We consider this to be a natural behavior because the value of the standard deviation decreases as the average sample size increases for a specific series. Besides, it is observed that for the acquisition frequency of $1 \mathrm{KHz}$ the standard deviation increases

as the resistance of the cell increases. However, for this acquisition frequency, the $\sigma \mathrm{i}$ varies from $140 \mathrm{pA}$ for the $100 \Omega$ dummy cell to $200 \mathrm{pA}$ for the $1 \mathrm{M} \Omega$ dummy cell, the $\sigma \mathrm{v}$ varies from $120 \mu \mathrm{V}$ for the $100 \Omega$ dummy cell to $200 \mu \mathrm{V}$ for the $1 \mathrm{M} \Omega$ dummy cell. Although the values obtained for the $\sigma \mathrm{i}$ and $\sigma \mathrm{V}$ are higher than $\sigma \mathrm{t}$ obtained for the calculated thermal noise of the resistance, which is reasonable because these thermal noise values are very small, these values are in the range established by the ASTM G199. ${ }^{13}$

Figures $11 \& 12$ show the latest two and half hours of the variation in current noise and potential noise overtime of a piece of aluminum which was immersed six hours in a solution of distilled water with $3.5 \%$ of sodium chloride. These figures used an acquisition frequency of $1 \mathrm{KHz}$ as Figures 5 to 10 . Measurement started after four hours to allow $\mathrm{NaCl}$ to modify the physical properties of the outer passive layer of aluminum that allows ion exchange. However, after that period, the current noise time series presents very short peaks which are related to localized corrosion processes and specifically pitting corrosion. 
Table 2 The average standard deviation for the current $(\sigma i)$ and potential $(\sigma \mathrm{v})$ time records and the thermal noise of the resistance $(\sigma \mathrm{t})$ at three different acquisition frequency and each dummy cell.

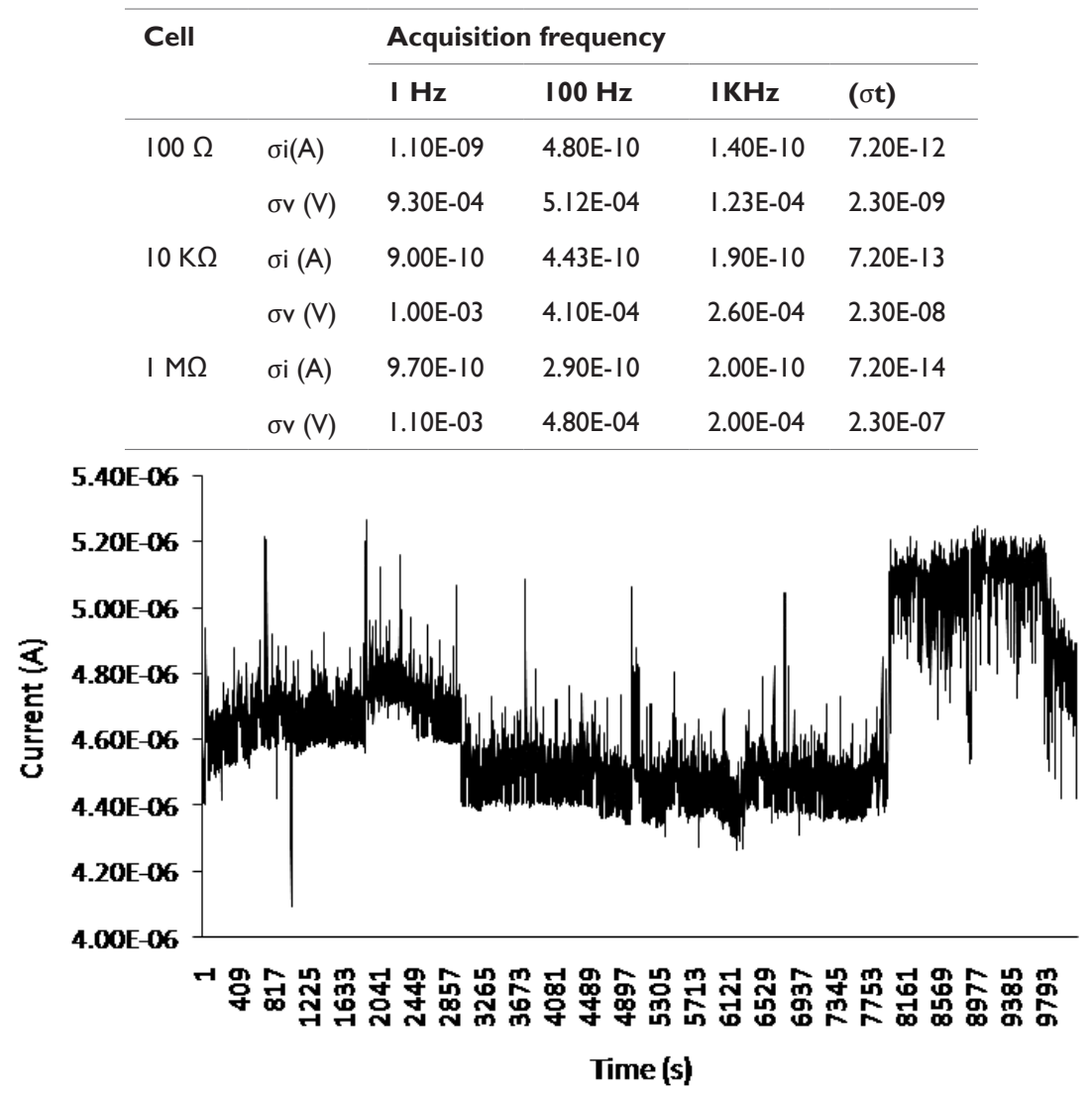

Figure I I Temporal behavior of current noise of aluminum.

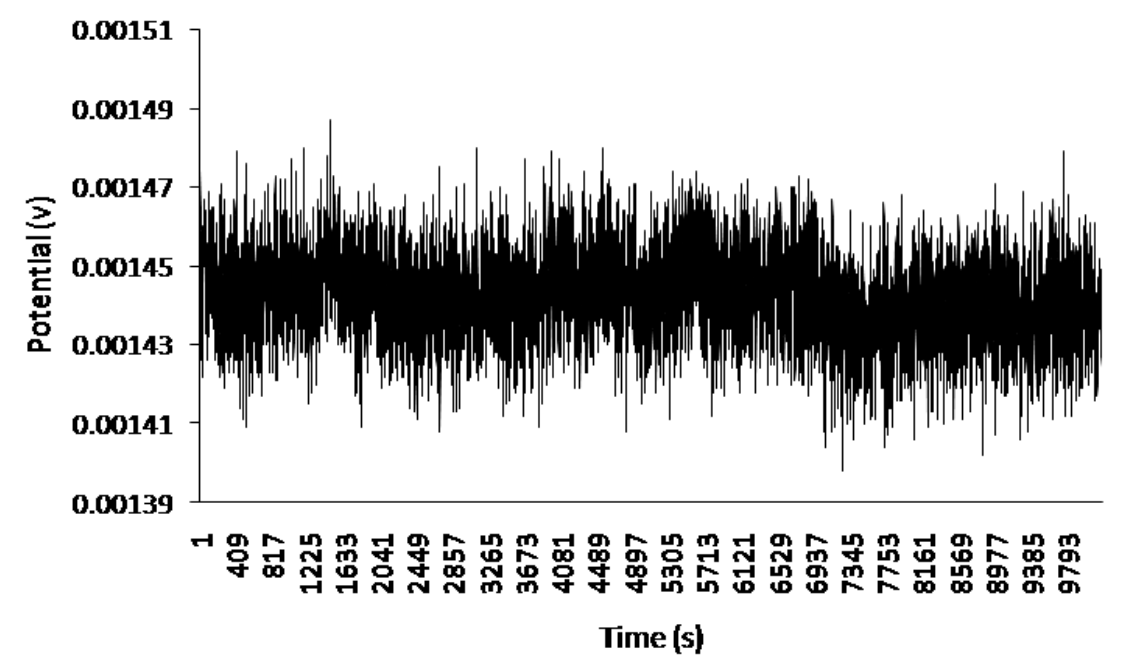

Figure 12 Temporal behavior of the potential noise of aluminum.

The current noise time series shows an increase in its baseline from 4.4 to $5 \mu \mathrm{A}$. This increase in current noise reflects an increase in the rate of corrosion as reported in. ${ }^{4,5}$ However, the potential noise time series shows an increase in its baseline of only $150 \mu \mathrm{V}$ that validates the stability of the system.

On the other hand, to get the information about the kinetic reaction and to confirm the type of corrosion that is occurring we use the pitting index $(\mathrm{PI})^{5}$ (see Table 1). Figure 13 shows the behavior of PI in the latest two and a half hours of the experiment.

Finally, the analysis of the data of Figure 13 shows the type of corrosion present in the piece of aluminum, after four hours immersed in $3.5 \%$ of $\mathrm{NaCl}$ solution, is mixed because most values of the PI are in the range between 0.01 and 0.1 and in the last half hour the main type of corrosion is associated to localized corrosion. 


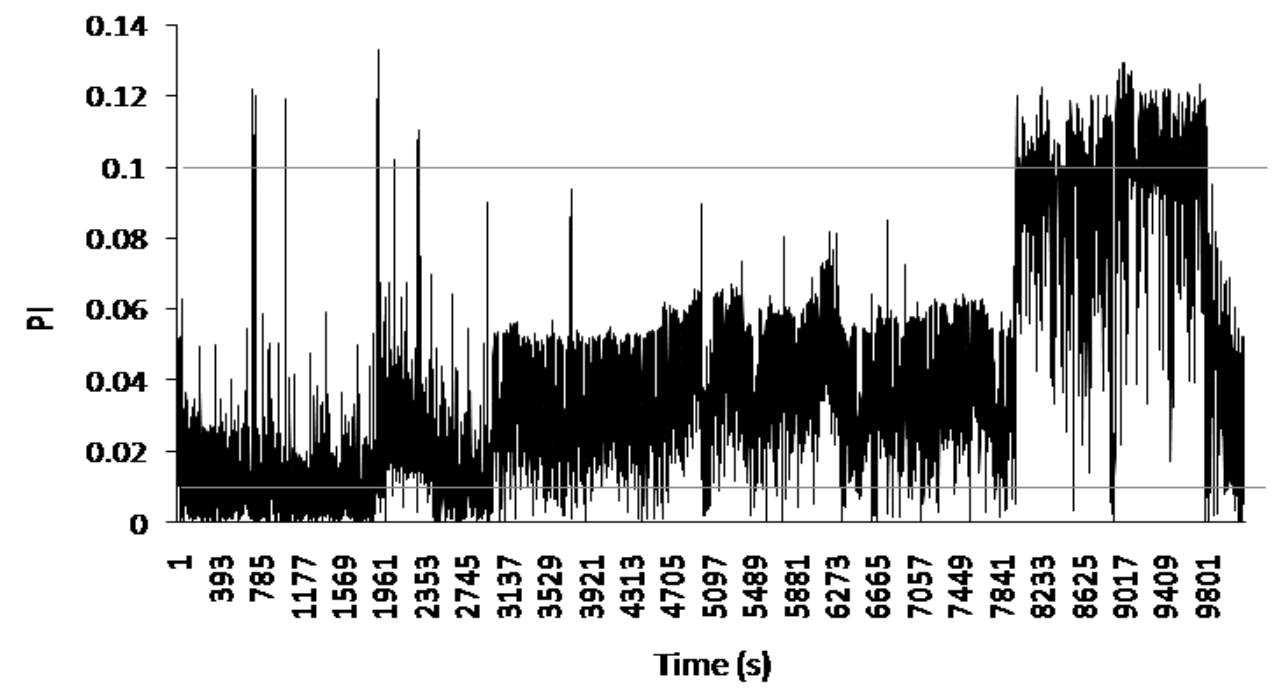

Figure 13 Pitting index behavior.

\section{Conclusion}

In this paper, we show the development of a low-cost electronic system for the measurement of electrochemical noise to identify the existence of corrosion and its type.

We carried out several tests using three different resistive cells and three different acquisition frequencies. Those tests showed that the better results were obtained for the acquisition frequency of $1 \mathrm{KHz}$ in which the $\sigma$ i varies from $140 \mathrm{pA}$ for the $100 \Omega$ dummy cell to $200 \mathrm{pA}$ for the $1 \mathrm{M} \Omega$ dummy cell, the $\sigma \mathrm{V}$ varies from $120 \mu \mathrm{V}$ for the $100 \Omega$ dummy cell to $200 \mu \mathrm{V}$ for the $1 \mathrm{M} \Omega$ dummy cell, which demonstrates the low level of intrinsic noise of the developed system. Although the values obtained for the $\sigma \mathrm{i}$ and $\sigma \mathrm{v}$ are higher than $\sigma \mathrm{t}$, obtained for the calculated thermal noise of the resistance there are in the range established by the ASTM G199 for the ENM systems.

Furthermore, the measurements for a piece of aluminum immersed in a solution of $3.5 \%$ of $\mathrm{NaCl}$ were accurate, showing a localized corrosion in the form of pitting that characterizing this type of experiment.

Therefore, a reliable and low-cost electronic system was developed, which since its technical characteristics allows online measurements electrochemical noise in metals exposed to electrolyte with resistive below $1 \mathrm{M} \Omega$ and in different environments.

\section{Acknowledgments}

This work was supported by the Autonomous University of Tamaulipas and National Council for Science and Technology (CONACYT), Mexico, scholarship number 480573.

\section{Conflicts of interest}

The authors declare that there is no conflict of interest.

\section{References}

1. Eden DA, Meng QJ, Méndez M, et al. Electrochemical noise. In: Revie RW. editor. Uhlig's Corrosion Handbook, 3rd ed. John Wiley \& Sons, Inc,Hoboken NJ, USA. 2011;1167e1177.
2. Iverson WP. Transient voltage changes produced in corroding metals and alloys. In: Fort Detrick FM. ditor. J Electrochem Soc. 1968;115(6).

3. RA Cottis. Inter pretation of electrochemical noise data. Corrosion. 2001;57(3):265-285.

4. Rik-Wouter Boscha, Robert A Cottis, Kinga Csecs, et al. Reliability of electrochemical noise measurements: Results ofround-robin testing on electrochemical noise. Electrochimica Acta. 2014;120:379-389.

5. JH Arellano-Pérez, OJ. Ramos-Negrón, RF Escobar-Jiménez, et al Development of a portable device for measuring the corrosion rates of metals based on electro chemical noise signals. Measurement. 2018;122:73-81.

6. S Reid, D Eden. Assessment of corrosion, US Patent 6,264,824 B1 (Jul. 24, 2001).

7. JAR Enciso, AR Salinas. Sistema que supervisa la corrosión en tiempo real por análisis del ruido electroquímico. Scientia et Technica. Año XIII. 2007;(36):937-940.

8. Bihade MS, Patil AP, Khobragade NK. Analysis of electrochemical current noise from meta stable pitting of SS304L in NaCl solutions. Trans Indian Inst Met. 2013;66;155-161.

9. Girija S, Mudali UK, Raju VR, et al. Determination of corrosion types for AISI type 304L stain less steel usingelectrochemical noise method. Mater Sci Eng. A.2005;407(1-2):188-195.

10. Bertocci U, Huet F, Nogueira RP. Use of multiple reference electrodes in electrochemical noise measurements. Corrosion. 2003;59(7):629e634.

11. Brusamarello V, Lago A, Franco CV. Analysis of different methods toncalculate electrochemical noise resistance using a three- electrode cell. Corrosion. 2000;56(3):273-282.

12. Naing, NA, Wong KW, Tan Yong-Jun. A novel electrochemical method formonitoring corrosion under insulation. Anti-Corrosion Methods Mater. 2006;53(3):175-179.

13. ASTM G199-09. Standard guide for electrochemical noise measurement, in: Annual book of ASTM standards, ASTM International, West Conshohocken, PA, USA, 2014;03(02).

14. S Ritter, F Huet, RA Cottis. Guideline for an assessment of electrochemical noise measurement devices. Materials and Corrosion. 2012;63(4):297302 . 